\title{
RIGID MONOMIAL IDEALS
}

\author{
TIMOTHY B.P. CLARK AND SONJA MAPES
}

\begin{abstract}
In this paper we investigate the class of rigid monomial ideals and characterize them by the fact that their minimal resolution has a unique $\mathbf{Z}^{d}$-graded basis. Furthermore, we show that certain rigid monomial ideals are lattice-linear, so their minimal resolution can be constructed as a poset resolution. We then give a description of the minimal resolution of a larger class of rigid monomial ideals by appealing to the structure of $\mathcal{L}(n)$, the lattice of all lcmlattices of monomial ideals on $n$ generators. By fixing a stratum in $\mathcal{L}(n)$ where all ideals have the same total Betti numbers, we show that rigidity is a property which propagates upward in $\mathcal{L}(n)$. This allows the minimal resolution of any rigid ideal contained in a fixed stratum to be constructed by relabeling the resolution of a rigid monomial ideal whose resolution has been constructed by other methods.
\end{abstract}

Introduction. Giving a construction for the minimal free resolution of a monomial ideal is a question which has motivated a wide variety of projects in commutative algebra. Various methods are known for computing the $\mathbf{Z}^{d}$-graded Betti numbers in the general case, and there are numerous strategies for constructing maps in free resolutions. Despite this diversity of results, it is still not known how to construct the maps in a minimal $\mathbf{Z}^{d}$-graded free resolution, except for several subclasses of monomial ideals (generic and Borel are examples).

In this paper we explore the class of rigid monomial ideals which were introduced to us by Ezra Miller [10]. By definition, a rigid ideal has the following two properties; (R1) every nonzero $\mathbf{Z}^{d}$-graded Betti number equals one, and (R2) $\mathbf{Z}^{d}$-graded Betti numbers which are nonzero in the same homological degree correspond to incomparable monomials in the lcm-lattice of the ideal. Generic monomial ideals and monomial ideals whose minimal resolution is supported on their Scarf complex [1] are two well-studied subclasses of rigid ideals. Furthermore, rigid ideals are a generalization of the class of monomial ideals in three variables

Received by the editors on March 10, 2011, and in revised form on September 20, 2012. 
studied by Miller in [9], who constructed their minimal resolution using a rigid embedding into a grid surface.

The first result presented herein is Theorem 1.1, which was communicated [10] along with Definition 1.1. This characterizes rigid monomial ideals as having a minimal resolution with unique $\mathbf{Z}^{d}$-graded basis. As such, the $\mathbf{Z}^{d}$-graded maps in such a minimal resolution should be explicitly describable. Our interest in studying rigid ideals stems from a desire to identify combinatorial objects which encode the data of these maps.

More precisely, since the $\mathbf{Z}^{d}$-graded Betti numbers of a monomial ideal can be computed from the lcm-lattice, it seems that a description of the maps in a rigid ideal's minimal resolution using the relations in the lcm-lattice should be possible. We aim toward this goal by taking advantage of the construction described by the first author in [5]. This construction takes as its input the lcm-lattice of a monomial ideal and produces an approximation to the minimal resolution of the ideal. In the case when the minimal resolution is indeed obtained, the ideal is said to be lattice-linear. More generally, if a $\mathbf{Z}^{d}$-graded poset is used as input for this construction and the resulting sequence is an exact complex of $\mathbf{Z}^{d}$-graded free modules, it is called a poset resolution.

In this paper we focus attention on a subclass of rigid ideals which we call concentrated. An ideal is said to be concentrated if the monomials corresponding to the $\mathbf{Z}^{d}$-degrees of basis elements in the minimal resolution are less than, or incomparable to, those monomials in the lcm-lattice which do not contribute a basis element to the minimal resolution. Theorem 2.1 states that a concentrated rigid monomial ideal is lattice-linear, which allows the construction of a minimal poset resolution for any concentrated rigid monomial ideal.

Next, we develop a method for transferring the resolution information of a rigid monomial ideal to related rigid ideals which have the same total Betti numbers. In particular, we consider rigid ideals in relation to their neighbors in $\mathcal{L}(n)$, the set of all finite atomic lattices (or lcm-lattices) with $n$ ordered atoms. In [14], Theorem 4.2 shows that under an appropriate partial order, $\mathcal{L}(n)$ is itself a finite atomic lattice. Furthermore, Theorem 3.3 in [7] implies that total Betti numbers increase along chains in $\mathcal{L}(n)$. It is therefore natural to study the 
subposets of $\mathcal{L}(n)$ with fixed total Betti numbers, which we refer to individually as Betti stratum. Within a fixed Betti stratum, we classify certain families of monomial ideals with non-isomorphic lcm-lattices which have minimal resolutions that are the same up to relabeling. Precisely, Theorem 3.2 states that any two comparable rigid monomial ideals in a fixed Betti stratum must have minimal resolutions which are relabelings of one another. This allows us to extend Theorem 2.1 and construct a minimal resolution for a larger subclass of rigid monomial ideals. We close the paper by briefly viewing rigid ideals through the lens of known topological construction techniques for resolutions.

The notion of a partially ordered set (poset), its order complex, and related notions from combinatorial algebraic topology are assumed to be familiar to the reader.

1. Rigid monomial ideals. Let $R=\mathbf{k}\left[x_{1}, \ldots, x_{d}\right]$, taken with the standard $\mathbf{Z}^{d}$ grading, where $\mathbf{k}$ is a field. We write $\mathbf{x}^{\mathbf{b}}=x_{1}^{b_{1}} \cdots x_{d}^{b_{d}}$ for a monomial in $R$ and refer to $\mathbf{b}=\left(b_{1}, \cdots, b_{d}\right)$ as the $\mathbf{Z}^{d}$-degree of the monomial $\mathbf{x}^{\mathbf{b}}$, which we write $\operatorname{mdeg}\left(\mathbf{x}^{\mathbf{b}}\right)$. When the context is clear, we abuse notation and refer to a monomial and its $\mathbf{Z}^{d}$-degree interchangeably. We consider only ideals $M \subset R$ which are generated by monomials and refer to $\mathcal{F}$, a minimal $\mathbf{Z}^{d}$-graded free resolution of $R / M$, simply as a minimal resolution.

Recall that for a monomial ideal $M$, the lcm-lattice $\mathbf{L C M}(M)$ is the set of least common multiples of the minimal generators $m_{1}, \ldots, m_{n} \in$ $M$. The monomials in $\mathbf{L C M}(M)$ are ordered by divisibility, the maximal element of $\mathbf{L C M}(M)$ is $\operatorname{lcm}\left(m_{1}, \ldots, m_{n}\right)$, and the minimal element is 1 (considered as the lcm of the empty set of monomials). In the case where $m<m^{\prime} \in \mathbf{L C M}(M)$ and there is no monomial $m^{\prime \prime} \in \mathbf{L C M}(M)$ such that $m<m^{\prime \prime}<m^{\prime}$, we say that $m^{\prime}$ covers $m$ and write $m \lessdot m^{\prime}$. Throughout the paper, the notation $\lessdot$ is used in the same way when considering the coverings in any poset.

Gasharov, Peeva and Welker [7] first defined the lcm-lattice and derived a formula for the $\mathbf{Z}^{d}$-graded Betti numbers $\beta_{i, \mathbf{b}}$ of $M$ based on the homology of its open intervals. Furthermore, a result of Bruns and Herzog [4, Theorem 3.1a], guarantees that if a $\mathbf{Z}^{d}$-graded Betti number $\beta_{i, \mathbf{b}}$ is nonzero, then the monomial $\mathbf{x}^{\mathbf{b}} \in \mathbf{L C M}(M)$. 

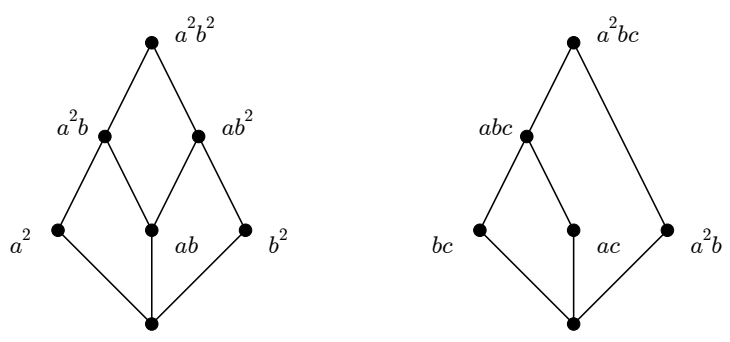

Figure 1. The lcm-lattices of the rigid monomial ideal $M$ and the non-rigid monomial ideal $N$.

With an eye on the lcm-lattice, we turn to the class of monomial ideals which are the main topic of study for this paper. The following definition was communicated [10] to the second author by Miller.

Definition 1.1. A monomial ideal $M$ is a rigid monomial ideal if its $\mathbf{Z}^{d}$-graded Betti numbers satisfy the following two properties:

(R1) $\beta_{i, \mathbf{b}}$ is either 1 or 0 for all $i$ and all $\mathbf{b} \in \mathbf{Z}^{d}$.

(R2) If $\beta_{i, \mathbf{b}}=1$ and $\beta_{i, \mathbf{b}^{\prime}}=1$ then $\mathbf{x}^{\mathbf{b}}$ and $\mathbf{x}^{\mathbf{b}^{\prime}}$ are incomparable in $\operatorname{LCM}(M)$.

Note that since ( $\mathbf{Z}^{d}$-graded) Betti numbers are dependent on the characteristic of the field $\mathbf{k}$, the condition of an ideal being rigid is as well. In fact, the well-known example of the monomial ideal arising from a triangulation of the real projective plane is rigid in characteristic other than 2 and not rigid when the characteristic is 2 .

Example 1.1. The monomial ideal $M=\left(a^{2}, a b, b^{2}\right)$ is rigid since the Betti numbers $\beta_{1, a^{2} b}=\beta_{1, a b^{2}}=1$, but $a^{2} b$ and $a b^{2}$ are incomparable monomials in $\mathbf{L C M}(M)$. The monomial ideal $N=\left(b c, a c, a^{2} b\right)$ is not rigid since the Betti numbers $\beta_{1, a b c}=\beta_{1, a^{2} b c}=1$, but $a b c$ and $a^{2} b c$ are comparable monomials in $\mathbf{L C M}(N)$. These lcm-lattices are shown in Figure 1.

For a rigid ideal, the existence of a nonzero Betti number in $\mathbf{Z}^{d_{-}}$ degree $\mathbf{b}$ precludes the existence of another nonzero Betti number in $\mathbf{Z}^{d}$-degree $\mathbf{b}$ from appearing in a different homological degree. 
Corollary 1.2. Let $M$ be a rigid monomial ideal. If $\beta_{i, \mathbf{b}}(R / M)=1$ then $\beta_{j, \mathbf{b}}(R / M)=0$ for all $j \neq i$.

Proof. For any monomial ideal, if $i=1$ then $\mathbf{b}$ corresponds to $\mathbf{x}^{\mathbf{b}}, \mathbf{a}$ minimal generator of the ideal. The monomial $\mathbf{x}^{\mathbf{b}}$ therefore only covers the monomial 1 in the lcm-lattice, so that the order complex $\Delta\left(1, \mathbf{x}^{\mathbf{b}}\right)$ is the empty simplicial complex. Hence, using the formula for $\mathbf{Z}^{d}$-graded Betti numbers in [7], we have $\beta_{j, \mathbf{b}}=0$ for $j \neq 1$.

Next, let $i>1$ and assume that $M$ is a rigid monomial ideal with minimal resolution $\mathcal{F}$. Suppose that there exists $j>i$ with the property that $\beta_{j, \mathbf{b}}=1$. The $\mathbf{Z}^{d}$-graded strand $\mathcal{F}_{\leq \mathbf{b}}$ therefore extends to at least homological degree $j$. This means that $\mathcal{F}_{\leq \mathbf{b}}$ contains free modules in homological degree $i$ so there must exist $\mathbf{a} \in \mathbf{Z}^{d}$ such that $\mathbf{x}^{\mathbf{a}}<\mathbf{x}^{\mathbf{b}}$, but $\beta_{i, \mathbf{a}}=1$. This contradicts condition (R2) in Definition 1.1.

Assuming the reverse inequality $j<i$ and modifying the above argument also produces the same contradiction to condition (R2). Hence, such a $j$ cannot exist and $\beta_{j, \mathbf{b}}(R / M)=0$ for all $j \neq i$ when $M$ is rigid.

Our main interest in studying rigid monomial ideals follows from the existence of a unique $\mathbf{Z}^{d}$-graded basis in their minimal free resolution, which was communicated to the second author by Miller [10]. We restate this result with proof since it does not appear in the literature.

Remark 1.1. When referring to a unique $\mathbf{Z}^{d}$-graded basis, we assume a fixed monomial ordering and imply that this basis is unique with respect to this monomial ordering. Furthermore, any scaling of $\mathbf{Z}^{d_{-}}$ graded basis elements by scalars of $R$ does not affect the underlying $\mathbf{Z}^{d}$-grading. Hence, when we use the term unique basis to mean unique up to scaling by elements in $\mathbf{k}$.

Proposition 1.1. Let $M$ be a monomial ideal. The minimal resolution of $R / M$ has a unique $\mathbf{Z}^{d}$-graded basis if and only if $M$ is a rigid monomial ideal.

We note that in particular, every $\mathbf{Z}^{d}$-degree which appears in the resolution of a rigid ideal does so as the $\mathbf{Z}^{d}$-degree of a basis element for exactly one free module of rank one. Furthermore, when considering 
the minimal resolution of a rigid ideal with respect to a fixed monomial ordering, any minimal resolution corresponding to a different monomial ordering may be obtained by permuting this unique $\mathbf{Z}^{d}$-graded basis. This structure gives hope that the minimal resolution of a rigid ideal is supported on a unique combinatorial object.

In order to prove Proposition 1.1, we first establish the following technical result.

Lemma 1.1. Fix $i>0$ and suppose that we have chosen $\mathbf{Z}^{d}$-graded bases for the free modules of the minimal resolution of $R / M$ in every homological degree less than $i$.

There is a unique choice of $\mathbf{Z}^{d}$-graded basis for the free $R$-module $F_{i}$ if and only if $\beta_{i, \mathbf{b}}=1$ or 0 for all $\mathbf{b} \in \mathbf{Z}^{d}$ and $\beta_{i, \mathbf{b}}=\beta_{i, \mathbf{b}^{\prime}}=1$ implies that $\mathbf{b}$ and $\mathbf{b}^{\prime}$ are not comparable in $\mathbf{L C M}(M)$.

Note that the conditions in Lemma 1.1 are relaxed instances of the conditions in the definition of a rigid monomial ideal, as they only hold for a specific $i$. In the case when $M$ is rigid, then the conditions on Betti numbers described in Lemma 1.1 hold for all $i$.

Proof of Lemma 1.1. $(\Leftarrow)$. Suppose that the conditions on $\mathbf{Z}^{d}$-graded Betti numbers hold and let $\left\{e_{1}^{i}, \cdots, e_{\beta_{i}}^{i}\right\}$ and $\left\{f_{1}^{i}, \cdots, f_{\beta_{i}}^{i}\right\}$ be distinct $\mathbf{Z}^{d}$-graded bases of $F_{i}$, the free module of rank $\beta_{i}$ appearing in the minimal resolution. For every $1 \leq \ell \leq \beta_{i}$ we may write

$$
f_{\ell}^{i}=\sum_{j=1}^{\beta_{i}} n_{j}^{i} \cdot e_{j}^{i}
$$

in terms of the basis $\left\{e_{1}^{i}, \cdots, e_{\beta_{i}}^{i}\right\}$ where $n_{j}^{i} \in R$. Preservation of $\mathbf{Z}^{d_{-}}$ degrees under this change of basis guarantees $\operatorname{mdeg}\left(f_{\ell}^{i}\right)=\operatorname{mdeg}\left(n_{j}^{i}\right)+$ $\operatorname{mdeg}\left(e_{j}^{i}\right)$ for every $j$ and as such, $\mathbf{x}^{\operatorname{mdeg}\left(e_{j}^{i}\right)} \leq \mathbf{x}^{\operatorname{mdeg}\left(f_{\ell}^{i}\right)} \in \mathbf{L C M}(M)$ for those $e_{j}^{i}$ with the property that $n_{j}^{i} \neq 0$. Unless $\mathbf{x}^{\operatorname{mdeg}\left(e_{j}^{i}\right)}=\mathbf{x}^{\operatorname{mdeg}\left(f_{\ell}^{i}\right)}$, this comparability in $\mathbf{L C M}(M)$ contradicts the hypothesis that if $\beta_{i, \mathbf{b}}=$ $\beta_{i, \mathbf{b}^{\prime}}=1$ then $\mathbf{b}$ and $\mathbf{b}^{\prime}$ are not comparable. When equality holds, the assumption that $\beta_{i, \mathbf{b}}=1$ or 0 implies that $n_{j}^{i}=0$ for all but one $j$. Hence, $f_{\ell}^{i}=n_{j}^{i} \cdot e_{j}^{i}$ for some $j$, which forces $\operatorname{mdeg}\left(n_{j}^{i}\right)=\mathbf{0}$. Thus, $f_{\ell}^{i}$ is a scalar multiple of a unique $e_{j}^{i}$ for every $\ell$, proving the uniqueness of $\mathbf{Z}^{d}$-graded basis for $F_{i}$. 
( $\Rightarrow$, by contrapositive). If there is not a unique $\mathbf{Z}^{d}$-graded basis for $F_{i}$ then we will show that one of the conditions fails.

Suppose that there are distinct $\mathbf{Z}^{d}$-graded bases so that using the notation established above, there exists $\ell$ such that

$$
f_{\ell}^{i}=\sum_{j=1}^{\beta_{i}} n_{j}^{i} \cdot e_{j}^{i},
$$

where at least two of the $n_{j}^{i}$ are nonzero. Write $\mathbf{f}$ for the $\mathbf{Z}^{d}$-degree of $f_{\ell}^{i}$. If two or more of the $e_{j}^{i}$ appearing in the expansion are of $\mathbf{Z}^{d}$-degree $\mathbf{f}$, then $\beta_{i, \mathbf{f}}>1$, so that the first condition fails. On the other hand, if the first condition holds, and $\mathbf{f}$ is the $\mathbf{Z}^{d}$-degree of any one of the $e_{j}^{i}$ in the expansion, then there is another nonzero term of $\mathbf{Z}^{d}$-degree $\mathbf{e} \neq \mathbf{f}$ having the property that $\mathbf{x}^{\mathbf{f}}>\mathbf{x}^{\mathbf{e}} \in \mathbf{L C M}(M)$. This causes the failure of the second condition. If the linear combination does not include basis elements of $\mathbf{Z}^{d}$-degree $\mathbf{f}$, then the second condition fails, for there would be nonzero Betti numbers in the same homological degree corresponding to comparable monomials in the lcm-lattice.

Remark 1.2. Note that in the proof of Lemma 1.1, we do not explicitly use the bases of the modules $F_{j}$ where $j<i$. Since we show that the basis of $F_{i}$ is unique after making choices in a lower homological degree, it is unnecessary to use the bases of $F_{j}$ in the proof. We give an example of a non-rigid ideal that demonstrates the conclusion of the lemma, even though the basis for one of the $F_{i}$ is not unique.

Consider the ideal $J=\left\langle a b e f, c d e f, b^{2} \operatorname{def}, a b^{2} d f, a^{2} c e f, a^{2} b c e\right\rangle$ in the $\operatorname{ring} R=\mathbf{k}[a, \cdots, f]$. The ideal $J$ is not rigid, but satisfies the hypotheses of Lemma 1.1 for $i=3$, since $\beta_{3, a b^{2} c d e f}=\beta_{3, a^{2} b c d e f}=1$. There are several choices for the basis elements of $F_{2}$, each of which forces a unique choice of basis for $F_{3}$. For example, for the monomial $a b^{2} d e f$, we have $\beta_{2, a b^{2} \text { def }}=2$, so one can choose any two of the elements from the set

$$
\{(b d, 0,-a, 0,0,0),(b d, 0,0,-e, 0,0),(0,0, a,-e, 0,0)\}
$$

to form a basis. Choosing the second and third elements corresponds to a unique choice of basis for $F_{3}$, whose $\mathbf{Z}^{d}$-degree is $a b^{2} c d e f$. Either of the other two choices give rise to a different (but unique) choice for $\mathbf{Z}^{d}$-degree $a b^{2}$ cdef basis element of $F_{3}$. 
Proof of Proposition 1.1. Let $M$ be an ideal in $R$ minimally generated by $q$ monomials and write $\mathcal{F}$ for the minimal resolution of $R / M$.

The minimal resolution $\mathcal{F}$ has $F_{0}=R$, a free module of rank one, appearing in homological degree zero. This module has a unique basis element of $\mathbf{Z}^{d}$-degree $\mathbf{0}=(0, \ldots, 0)$.

Since the $q$ minimal generators of a monomial ideal are unique, the free module $F_{1}$ is of rank $q$ and has basis elements which are $\mathbf{Z}^{d}$-graded to match the $\mathbf{Z}^{d}$-degrees of the monomial generators. Any choice of basis for $F_{1}$ which corresponds to an $R$-linear combination of these generators gives a non-monomial generating set for the monomial ideal $M$. As we have not yet assumed rigidity, it follows that for any monomial ideal, the modules $F_{0}$ and $F_{1}$ in the minimal resolution have unique $\mathbf{Z}^{d}$-graded bases.

If the minimal resolution of $R / M$ has a unique $\mathbf{Z}^{d}$-graded basis, then Lemma 1.1 guarantees the conditions of rigidity for every $i>1$, and therefore, $M$ is a rigid monomial ideal.

Conversely, let $i>1$ and suppose that $M$ is a rigid monomial ideal. We proceed by strong induction. Suppose that there is a unique choice of $\mathbf{Z}^{d}$-graded basis for each free module $F_{j}$, where $j<i$. This is a special case of the assumptions in Lemma 1.1, so that we are guaranteed a unique choice of $\mathbf{Z}^{d}$-graded basis for $F_{i}$. Hence, each free module in $\mathcal{F}$ has a unique $\mathbf{Z}^{d}$-graded basis.

2. $\mathbf{Z}^{d}$-graded minimal poset resolutions. The benefit of looking at rigid monomial ideals instead of the entire class of monomial ideals is that the unique $\mathbf{Z}^{d}$-graded basis gives a hope of writing down a closed form description of the minimal resolution.

We aim at this goal by introducing a notion that describes the $\mathbf{Z}^{d}$ degrees contributing to the minimal resolution in the context of the order relations of the lcm-lattice. We call a monomial ideal $M$ concentrated if every $\mathbf{Z}^{d}$-degree from $\mathbf{L C M}(M)$ which does not contribute a free module to the minimal resolution appears in a non-obstructive way in relation to all the contributing $\mathbf{Z}^{d}$-degrees in $\mathbf{L C M}(M)$. Precisely, every $\mathbf{Z}^{d}$-degree which is smaller than a contributing $\mathbf{Z}^{d}$-degree must itself contribute. Formally, we have the following. 
Definition 2.1. A monomial ideal $M$ is said to be concentrated if it has the property that for every $\mathbf{x}^{\mathbf{a}}$ such that $\beta_{j, \mathbf{a}}(R / M)=0$ for all $j$ then $\mathbf{x}^{\mathbf{a}} \nless \mathbf{x}^{\mathbf{b}} \in \mathbf{L C M}(M)$ for any $\mathbf{x}^{\mathbf{b}} \in \mathbf{L C M}(M)$ which has $\beta_{i, \mathbf{b}}(R / M) \neq 0$ for some $i$. A monomial ideal which is not concentrated is said to be dispersed.

The class of lattice-linear monomial ideals, whose minimal resolution was constructed in [5], consists of those monomial ideals whose lcmlattice serves as combinatorial support for the mapping structure in their minimal free resolution.

Definition 2.2. A monomial ideal $M$ is lattice-linear if $\mathbf{Z}^{d \text {-graded }}$ bases $B_{k}$ of the free modules $F_{k}$ appearing in the minimal resolution of $R / M$ can be fixed for all $k$ so that for any $i \geq 1$ and any $b \in B_{i}$ the differential

$$
d_{i}(b)=\sum_{b^{\prime} \in B_{i-1}} m_{b, b^{\prime}} \cdot b^{\prime}
$$

has the property that if the coefficient $m_{b, b^{\prime}} \neq 0$ then $\mathbf{x}^{\mathbf{b}^{\prime}}$ is covered by $\mathbf{x}^{\mathbf{b}}$ in the lcm-lattice $\mathbf{L C M}(M)$.

The rigid concentrated monomial ideal $M$ in Example 1.1 is latticelinear, while the non-rigid ideal $N$ is not lattice-linear. More generally, the property of being concentrated does not characterize rigid monomial ideals which are lattice-linear. We have, however, the following result.

Theorem 2.1. If a rigid monomial ideal is concentrated, then it is lattice-linear.

Proof. (By contrapositive). Suppose that $M$ is a rigid monomial ideal which is not lattice-linear and write $(\mathcal{F}, \partial)$ for the minimal resolution of $M$. For each $\ell$, let $B_{\ell}$ be the unique choice of basis for the free module $F_{\ell}$ appearing in the resolution.

Our supposition that $M$ is not lattice-linear implies that for some $i$, there exists a basis element $c \in B_{i}$ of $\mathbf{Z}^{d}$-degree $\mathbf{c}$ whose expansion under the differential does not mirror the covering relations in $\mathbf{L C M}(M)$. Precisely, there exists some basis element $a$ of $\mathbf{Z}^{d}$-degree $\mathbf{a}$ with the property that the coefficient $m_{a, c}$ in the expansion of $\partial_{i}(c)$ is nonzero, but that $\mathbf{x}^{\mathbf{a}}$ is not covered by $\mathbf{x}^{\mathbf{c}}$ in $\mathbf{L C M}(M)$. Hence, there exists 
$\mathbf{x}^{\mathbf{b}} \in \mathbf{L C M}(M)$ such that $\mathbf{x}^{\mathbf{a}} \lesseqgtr \mathbf{x}^{\mathbf{b}} \lesseqgtr \mathbf{x}^{\mathbf{c}}$. This comparison guarantees the existence of $\mathbf{Z}^{d}$-graded strands within the free resolution $\mathcal{F}$ which have the following containment structure;

$$
\mathcal{F}_{\leq \mathbf{a}} \subset \mathcal{F}_{\leq \mathbf{b}} \subset \mathcal{F}_{\leq \mathbf{c}} .
$$

This containment of complexes is contradicted unless the degree $\mathbf{b}$ strand terminates in homological degree $i$ or $i-1$, for otherwise the degree $\mathbf{c}$ and $\mathbf{a}$ strands would not terminate in homological degrees $i-1$ and $i$, respectively.

If indeed, the degree $\mathbf{b}$ strand terminates in either homological degree $i$ or $i-1$, we have identified two $\mathbf{Z}^{d}$-degrees which contribute in the same homological degree but correspond to comparable monomials in $\mathbf{L C M}(M)$. This contradicts the property (R2) in the definition of rigidity. Hence, the strand $\mathcal{F}_{\leq \mathbf{b}}$ cannot terminate in any homological degree and therefore, no degree $\mathbf{b}$ strand can exist within $\mathcal{F}$. In other words, $\beta_{\ell, \mathbf{b}}(R / M)=0$ for every $\ell$, so that any rigid ideal which is not lattice-linear cannot be concentrated.

Example 2.1. The converse of Theorem 2.1 is false. For example, the monomial ideal $K=\langle$ defgh, befgh, cefgh, afh, abcdh,abcde $\rangle$ is latticelinear and rigid, but has a dispersed lcm-lattice due to the existence of the noncontributing element abcdefh $\lessdot a b c d e f g h \in \mathbf{L C M}(K)$.

3. Similarity of resolutions within a subset of $\mathcal{L}(n)$. Theorem 2.1 gives a way to construct minimal free resolutions for a small class of rigid monomial ideals. In this section, we extend this construction to write minimal resolutions for a larger class of rigid monomial ideals by giving a partial converse to Theorem 3.3 in [7]. Central to this discussion is a lattice of finite atomic lattices, first introduced by Phan [14].

Let $\mathcal{L}(n)$ be the set of all finite atomic lattices with $n$ ordered atoms. Phan defines a partial order on $\mathcal{L}(n)$ as $P \geq Q$ if there exists a join preserving map $f: P \rightarrow Q$ which is a bijection on atoms. Theorem 4.2 in [14] shows that under this partial order, $\mathcal{L}(n)$ is itself a finite atomic lattice. Moreover, Theorem 3.3 in [7] indicates that total Betti numbers weakly increase as one moves up chains in $\mathcal{L}(n)$. Furthermore, Proposition 5.1 in [14] shows that every finite atomic lattice is the lcm-lattice of some monomial ideal. Thus, $\mathcal{L}(n)$ can be thought of 
as the lattice of all monomial ideals with $n$ ordered generators up to equivalence of lcm-lattices.

From the work of Gasharov, Peeva, and Welker [7] we know that for a monomial $\mathbf{x}^{\mathbf{b}} \in \mathbf{L C M}(M)$ the $\mathbf{Z}^{d}$-graded Betti numbers in homological degree $i$ are:

$$
\beta_{i, \mathbf{b}}(R / M)=\operatorname{dim} \widetilde{H}_{i-2}\left(\Delta\left(\hat{0}, \mathbf{x}^{\mathbf{b}}\right), \mathbf{k}\right)
$$

where $\Delta\left(\hat{0}, \mathbf{x}^{\mathbf{b}}\right)$ is the order complex of the open interval $\left(\hat{0}, \mathbf{x}^{\mathbf{b}}\right) \subset$ $\operatorname{LCM}(M)$. Equivalently, if $p \in P$ where $P$ is a finite atomic lattice (and therefore is isomorphic to the lcm-lattice of some monomial ideal) then we write

$$
\beta_{i, p}(P)=\operatorname{dim} \widetilde{H}_{i-2}(\Delta(\hat{0}, p), \mathbf{k})
$$

Moreover, since rigid monomial ideals are defined by the behavior of their $\mathbf{Z}^{d}$-graded Betti numbers, we classify a finite atomic lattice as rigid (or not) using the properties of Definition 1.1. In this section, we interchangeably refer to the Betti numbers $\beta_{i, p}$ of a finite atomic lattice $P$ and the Betti numbers $\beta_{i, \mathbf{b}}$ of an associated monomial ideal.

Let $\beta_{i}=\sum \beta_{i, \mathbf{b}}$ be the total Betti number of the ideal $M$ in homological degree $i$ and write $\beta=\left(\beta_{0}, \beta_{1}, \ldots, \beta_{t}\right)$ for the vector of total Betti numbers. We focus on subposets of $\mathcal{L}(n)$ which consist of all finite atomic lattices having the same total Betti numbers and refer to these subposets as Betti strata, denoting them $\mathcal{L}(n)_{\beta}$ for a fixed $\beta$. Given a rigid monomial ideal $M$ with total Betti numbers $\beta$, we now examine the relationship between $M$ and ideals with lcm-lattices in $\mathcal{L}(n)_{\beta}$.

Proposition 3.1. Let $P, Q \in \mathcal{L}(n)_{\beta}$ for some $\beta$ such that $P<Q$. If $P$ is rigid then $Q$ is rigid.

Proof. We first prove the proposition in the case when $P \lessdot Q \in \mathcal{L}(n)_{\beta}$. See Figure 2 for a pictorial comparison of the relevant portions of these posets.

From Proposition 5.1 in [8] we know that if $Q$ covers $P$, then as a set, $Q=P \cup\{q\}$ for some $q$. Moreover, since $P$ is a finite atomic lattice, we know that this unique $q \in Q$ must be meet-irreducible (it is not the meet of any two elements in $P$ ). So there is a unique element $p^{\prime} \in Q$ 
which covers $q$. To show that $Q$ is rigid, we must verify conditions (R1) and (R2). Note that for all $p<q$ in $Q$, the interval $(\hat{0}, p)$ in $Q$ is identical to the interval $(\hat{0}, p)$ in $P$, leaving the associated Betti number unchanged. Similarly, the Betti number is unchanged for all elements which are not comparable to $q$.

For any $p>p^{\prime}$ in $Q$ (or $P$ for that matter), we claim that the associated Betti number is also unchanged. To see this, consider the join preserving bijection on atoms $Q \rightarrow P$. The fiber over a point $p \in P$ is $\{p\}$ for $p \neq p^{\prime}$. Restricting this map to the intervals $(\hat{0}, p)$ in $Q$ where $p \gtrless p^{\prime}$, we see that the fibers of this map are contractible. By Quillen's Fiber Theorem (see Theorem 10.5 in [3]) the order complexes of these intervals in the lattices $P$ and $Q$ are homotopy equivalent.

It is therefore only necessary to check the conditions of rigidity for $p^{\prime}$ and $q$ in $Q$. When considering Betti number computations relative to different lattices, we introduce the notation $\beta_{i, p}^{P}$ and $\beta_{i, p}^{Q}$ to indicate the Betti number in $P$ and $Q$, respectively. By hypothesis, the total Betti numbers of $P$ and $Q$ are the same, and for all other $p \in Q$ we have seen that $\beta_{i, p}^{Q}$ is the same as in $P$. Hence, if $\beta_{i, p^{\prime}}^{P}=0$ for all $i$ then $\beta_{i, p^{\prime}}^{Q}$ and $\beta_{i, q}^{Q}$ are both 0 . Otherwise, if $\beta_{i, p^{\prime}}^{P} \neq 0$ then either $\beta_{i, q}^{Q}$ or $\beta_{i, p^{\prime}}^{Q}$ is 1 , but not both. Thus, condition (R1) is satisfied.

To see that condition (R2) holds, we must verify the case where $\beta_{i, p^{\prime}}^{P}=1$ for some $i$. If $\beta_{i, p^{\prime}}^{Q}=1$ then since $P$ is rigid, condition (R2) is satisfied for $Q$ as well. Alternatively, if $\beta_{i, q}^{Q}=1$ and condition (R2) is not satisfied then there is some $p \in Q$ distinct from $p^{\prime}$ such that $\beta_{i, p}^{Q}=1$ and $q$ and $p$ are comparable in $Q$. By the above argument however, we know that $\beta_{i, p}^{P}=1$. Moreover, if $p$ and $q$ are comparable in $Q$ then $p$ must also be comparable to $p^{\prime}$ in $Q$. This implies that $p$ and $p^{\prime}$ are comparable in $P$, which contradicts the fact that $P$ is rigid. As such, (R2) must be satisfied for $Q$.

The general statement for $P<Q$ is easily verified by induction on the length of a chain connecting $P$ to $Q$ in $\mathcal{L}(n)_{\beta}$.

We now work to extend the result of Theorem 2.1 to construct the minimal resolution of dispersed rigid monomial ideals within a Betti stratum which are greater than a concentrated rigid monomial ideal appearing in the same stratum. We use frames and the $M$-homogenization of [13], and include a description here for the convenience of the reader. 


$$
P \lessdot Q
$$
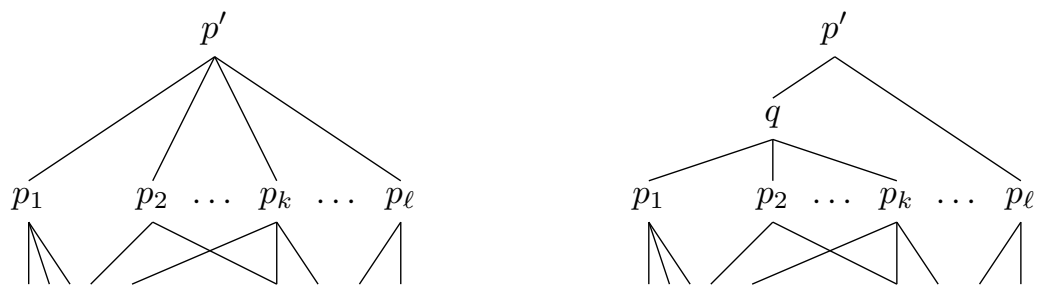

FiguRE 2. Relevant portions of the Hasse diagrams of the posets $P$ and $Q$ which are referred to in the proof of Proposition 3.1.

Definition 3.1. [13, Construction 3.1]. An $n$-frame is a complex $\mathcal{U}$ of finite $\mathbf{k}$-vector spaces with differential $\partial^{\mathcal{U}}$ and a fixed basis such that

(1) $U_{i}=0$ for all $i<0$ and all $i>>$,

(2) $U_{0}=\mathbf{k}$,

(3) $U_{1}=\mathbf{k}^{n}$, and

(4) $\partial^{\mathcal{U}}\left(w_{j}\right)=1$ for each basis vector $w_{j}$ in $U_{1}=\mathbf{k}^{n}$.

Let $\mathcal{F}$ be a resolution of of $R / M$ with a fixed $\mathbf{Z}^{d}$-graded basis where $M$ is a monomial ideal in a polynomial ring $R$. We find the frame of $\mathcal{F}$ by tensoring it with $R /\left(x_{1}-1, \ldots, x_{d}-1\right)$. Alternatively, to go from a frame to a complex of $R$-modules, we recall Construction 3.2 from [13].

Construction 3.1. Let $\mathcal{U}$ be an $n$-frame and $M$ be a monomial ideal with $n$ generators $\left\{m_{1}, \ldots, m_{n}\right\}$ in a polynomial ring $R$. The $M$ homogenization of $\mathcal{U}$ is a complex $\mathcal{F}$ of free $R$-modules with differential $\partial^{\mathcal{F}}$, which under certain conditions will be a resolution of $R / M$. Denote the $\mathbf{Z}^{d}$-degree of the monomial generator $m_{i}$ as $\mathbf{b}_{i}$ for each $1 \leq i \leq n$. First, define $F_{0}=R$ and $F_{1}=R\left(-\mathbf{b}_{1}\right) \oplus \cdots \oplus R\left(-\mathbf{b}_{n}\right)$.

Now, inductively define the remainder of the complex. Let $\bar{v}_{1}, \ldots \bar{v}_{e}$ and $\bar{u}_{1}, \ldots \bar{u}_{f}$ be the bases for $U_{i}$ and $U_{i-1}$ respectively. Let $u_{1}, \ldots, u_{f}$ denote the basis for $F_{i-1}=R^{f}$ (which we assume has been chosen in the previous step of the induction). Define in the following way the elements $v_{1}, \ldots, v_{e}$ which will be the basis for $F_{i}$. If $\partial\left(\bar{v}_{j}\right)=\sum_{1 \leq s \leq f} \alpha_{s j} \bar{u}_{s}$ 
then define the $\mathbf{Z}^{d}$-degree of $v_{j}$ to be $\operatorname{mdeg}\left(v_{j}\right)=\operatorname{lcm}\left(\operatorname{mdeg}\left(u_{s}\right) \mid \alpha_{s j} \neq\right.$ $0)$. Thus $F_{i}=\oplus_{1 \leq j \leq e} R\left(-\operatorname{mdeg}\left(v_{j}\right)\right)$ and the differential $\partial^{\mathcal{F}}\left(v_{j}\right)=$ $\sum_{1 \leq s \leq f} \alpha_{s j} \frac{\operatorname{mdeg}\left(v_{j}\right)}{\operatorname{mdeg}\left(u_{s}\right)} u_{s}$.

Remark 3.1. Let $P, Q$ be the lcm-lattices of monomial ideals $I_{P}$ and $I_{Q}$ in rings $R_{P}=k\left[x_{1}, \ldots, x_{d}\right]$ and $R_{Q}=k\left[y_{1}, \ldots, y_{d^{\prime}}\right]$ respectively. Note that by Theorem 4.13 in [13], if one starts with the minimal free resolution $\mathcal{F}_{Q}$ of $R_{Q} / I_{Q}$ and if $Q>P \in \mathcal{L}(n)$ then the relabeling construction (Construction 3.2 in [7]) is the $I_{P}$-homogenization of the frame of $\mathcal{F}_{Q}$.

Now, Corollary 4.15 in [13] gives a general version of a converse to Theorem 3.3 in [7]. In particular, if $Q>P$ in $\mathcal{L}(n)_{\beta}$ for a fixed $\beta$ then there exists a $\mathbf{Z}^{d}$-graded basis of a minimal free resolution $\mathcal{F}_{P}$ of $R_{P} / I_{P}$ such that the $I_{Q}$-homogenization of the frame of $\mathcal{F}_{P}$ is a minimal free resolution of $R_{Q} / I_{Q}$. The following proposition is a version of this result in the case when $I_{P}$ is rigid.

Proposition 3.2. Let $P$ be the lcm-lattice of monomial ideal $I_{P}$ in the polynomial ring $R_{P}$. If $I_{P}$ is rigid, then for any $Q>P$ in $\mathcal{L}(n)$, where $Q$ is the lcm-lattice of the monomial ideal $I_{Q}$ in the ring $R_{Q}$, the $I_{Q}$-homogenization of the frame of the minimal resolution $\mathcal{F}_{P}$ of $R_{P} / I_{P}$ is the minimal resolution of $R_{Q} / I_{Q}$.

Remark 3.2. The discussion in [13] suggests that in order to give a constructive method for minimal resolutions of monomial ideals, one first needs to answer the question of how to construct "good" frames. The main consequence of Proposition 3.2 is that when constructing "good" frames for rigid monomial ideals using their lcm-lattices, we can limit our focus to those rigid monomial ideals whose lcm-lattices are minimal in the sense that there are no rigid monomial ideals with smaller lcmlattices in the same Betti stratum. The new data of Proposition 3.2 is the underlying uniqueness of the $\mathbf{Z}^{d}$-graded basis for each resolution which results.

Proof of Proposition 3.2. By Corollary 4.15 in [13] we know that there exists a $\mathbf{Z}^{d}$-graded basis for the minimal free resolution $\mathcal{F}_{P}$ of $R_{P} / I_{P}$ 


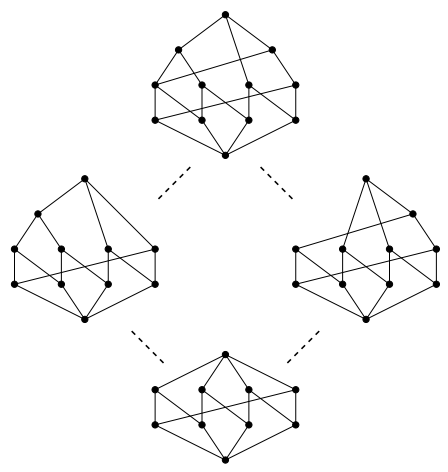

FiguRE 3. Unlabeled lcm-lattices of rigid monomial ideals.

such that the $I_{Q}$-homogenization of the frame of $\mathcal{F}_{P}$ is a minimal resolution of $R_{Q} / I_{Q}$. However, by Theorem 1.1 we know that since $I_{P}$ is rigid, there is a unique choice of $\mathbf{Z}^{d}$-graded basis for $\mathcal{F}_{P}$. This unique basis must be the basis guaranteed by Corollary 4.15 .

In the following example we see how Theorem 2.1 and Theorem 3.2 combine to construct minimal resolutions of certain rigid monomial ideals that are not concentrated.

Example 3.2. Figure 3 depicts unlabeled finite atomic lattices in the Betti stratum $\mathcal{L}(4)_{(1,4,4,1)}$. The lattice at the bottom of the figure is isomorphic to the lcm-lattice of a concentrated rigid monomial ideal, and therefore its minimal resolution can be constructed using Theorem 2.1.

The three lattices above the bottom lattice represent lcm-lattices of dispersed rigid monomial ideals, since the maximal element in all of the lattices corresponds to a single nonzero Betti number in homological degree 2. Theorem 3.2 guarantees that each of these ideals admit a minimal resolution whose maps mirror those of the concentrated rigid ideal pictured at the bottom of this interval in the stratum.

Remark 3.3. One can reinterpret Theorem 2.1 and Proposition 3.2 in the context of strata in $\mathcal{L}(n)$. Define $B(\mathbf{L C M}(M))$ to be the subposet of $\mathbf{L C M}(M)$ consisting of $\mathbf{x}^{\mathbf{b}} \in \mathbf{L C M}(M)$ such that $\beta_{i, \mathbf{b}}(R / M) \neq 0$ for some $i$, and call it the Betti poset of $\mathbf{L C M}(M)$. 
In the situation where $M$ is concentrated rigid, $B(\mathbf{L C M}(M))$ is the union of closed intervals $\left[\hat{0}, \mathbf{x}^{\mathbf{b}}\right] \subset \mathbf{L C M}(M)$ which have nonzero Betti numbers. Thus, one can compute the ranks of the free modules in the minimal free resolution by using either $\mathbf{L C M}(M)$ or $B(\mathbf{L C M}(M))$, as the pertinent information has not changed. Furthermore, if $M^{\prime}$ is an ideal such that $\mathbf{L C M}\left(M^{\prime}\right)>\mathbf{L C M}(M)$ within in the same Betti stratum, then Proposition 3.1 guarantees the rigidity of $M^{\prime}$. Therefore, the minimal free resolution of $M^{\prime}$ can be viewed as a relabeled poset resolution supported on $B(\mathbf{L C M}(M))$. Extensions of this approach are the subject of ongoing research.

4. Rigid ideals and topological resolutions. We now establish a connection between several well-studied topological resolutions and and the class of rigid monomial ideals. Recall the Scarf simplicial complex [1] associated to a monomial ideal $N$. This complex has its $n$ vertices in one-to-one correspondence with the monomial generators of $N$, and is defined as

$\Delta_{N}=\left\{I \subseteq\{1, \cdots, n\}: m_{I} \neq m_{J}\right.$ for all $J \subseteq\{1, \cdots, n\}$ other than $\left.I\right\}$, where $m_{I}=\operatorname{lcm}\left(m_{i}: i \in I\right)$. The ideal $N$ is called a Scarf ideal if its minimal free resolution is supported on the reduced simplicial chain complex of $\Delta_{N}$. For instance, generic ideals $[\mathbf{1}, \mathbf{1 1}]$ are Scarf.

Using the fact that the monomial label of any face $I \in \Delta_{N}$ is uniquely determined from $m_{I}$ as $I=\left\{i: m_{i}<m_{I}\right\}$, it is clear that each $\mathbf{Z}^{d}$-degree appears as the label of exactly one simplex, so that rigidity condition (R1) is satisfied for any Scarf monomial ideal. Suppose that rigidity condition (R2) does not hold for a Scarf ideal, so that there exist two simplices $I, J \in \Delta_{N}$ such that $m_{I}$ divides $m_{J}$. Considering the simplex $m_{I \cup J}$ within the full simplex on $n$ vertices, we see that $m_{I \cup J}=m_{J}$, contradicting the inclusion of the simplex $J$ in $\Delta_{N}$. We have therefore shown the following consequence of the definition of rigid monomial ideals.

\section{Corollary 4.1. Every Scarf ideal is a rigid monomial ideal.}

A generalization of this simplicial notion is the cellular resolution approach introduced in [2]. Using the language of frames, this approach can be described as using the augmented chain complex of a cell complex on $n$ vertices as an $n$-frame for a monomial ideal with $n$ 
generators. The following shows how some cellular resolutions can be understood through the lens of rigidity.

Proposition 4.1. Let $P_{X}$ be the augmented face poset of a regular $C W$ complex $X$. If $P_{X}$ is a finite atomic lattice and $X$ is acyclic, then $P_{X}$ is rigid. Moreover, if $Q>P_{X}$ in the stratum $\mathcal{L}(n)_{\beta}$, then $Q$ has a minimal resolution supported on $X$.

Proof. By Proposition 6.5 in [8] we know that a minimal resolution of the ideal whose lcm-lattice is $P_{X}$ is supported on $X$. Each cell $\alpha \in X$ contributes a unique $\mathbf{Z}^{d}$-degree, satisfying condition $(\mathrm{R} 1)$. Since no two cells of the same dimension are contained in one another, the noncomparability condition $(\mathrm{R} 2)$ is satisfied. Hence, $P_{X}$ is rigid. The second statement is a special case of Theorem 3.2.

Example 4.1. Let $E=\left(v_{1}, \ldots, v_{s}\right) \cap\left(w_{1}, \ldots, w_{t}\right)$ be the edge ideal of a complete bipartite graph, considered in the polynomial ring in $s+t$ variables, $\mathbf{k}\left[v_{1}, \ldots, v_{s}, w_{1}, \ldots, w_{t}\right]$. Visscher in [16] constructs a regular CW-ball which supports the (linear) minimal resolution of $E$. Furthermore, the face poset of this CW-ball is the lcm-lattice $\mathbf{L C M}(E)$ so that the edge ideal of a complete bipartite graph is a rigid, concentrated monomial ideal.

It is natural to ask whether every rigid ideal is minimally resolved on a (regular) CW-complex. However, even rigid ideals are too complicated to be resolved using purely topological methods. Recall the nearly Scarf ideals, introduced by Peeva and Velasco in [13]. The following result shows that certain ideals which do not admit topological resolutions are rigid, concentrated, and therefore lattice-linear. In particular, the example of a nearly Scarf ideal given by Velasco [15] which admits no CW resolution falls into this class.

Proposition 4.2. If $\Omega$ is a pure simplicial complex which is a homology sphere over $\mathbf{k}$, then the nearly Scarf monomial ideal $I_{\Omega}$ is rigid and concentrated.

Proof. By construction, any simplex of $\Omega$ has a unique $\mathbf{Z}^{d}$-degree, and no two simplices of the same dimension can be contained in one another. Hence, rigidity conditions (R1) and (R2) hold for the associated Betti 


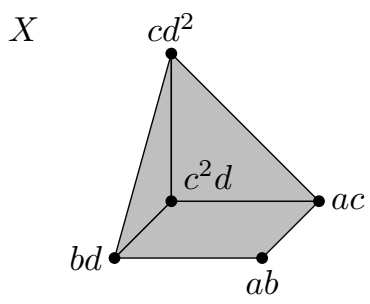

Figure 4. The regular CW-complex of Example 4.2, with monomial generators of $I$ labeling its vertices.

numbers. Since $\Omega$ is a pure homology sphere, the conditions for rigidity are trivially satisfied for the $\mathbf{Z}^{d}$-degree corresponding to a generator of the homology of $\Omega$. Furthermore, the lcm-lattice of $I_{\Omega}$ consists of the face poset of $\Omega$ with a top element adjoined. Hence, the lcmlattice has no noncontributing elements, guaranteeing that the ideal is concentrated.

We close the paper by providing an example of a rigid ideal whose minimal resolution is supported on a regular CW-complex, but which cannot be resolved by obtaining resolution information from concentrated rigid ideals appearing lower in the same Betti stratum. Indeed, for a given ideal, a comparable concentrated rigid ideal need not exist. We are unaware of conditions which guarantee the existence of such an ideal.

Example 4.2. The rigid monomial ideal $I=\left\langle b d, c d^{2}, a c, c^{2} d, a b\right\rangle$ is minimally resolved on the three-dimensional regular CW complex $X$, pictured in Figure 4 . Note that $X$ has $(1,5,7,4,1)$ as its face vector.

The face poset $P_{X}$ is not a lattice since the quadrilateral 2-cells on the sets of vertices $\left\{a b, a c, b d, c^{2} d\right\}$ and $\left\{a b, a c, b d, c d^{2}\right\}$ intersect in the union of 1-cells $\{a b, a c\} \cup\{a b, b d\}$. Therefore, $P_{X}$ is not contained in any Betti stratum of $\mathcal{L}(5)$. In fact, the lcm-lattice $\mathbf{L C M}(I)$ is isomorphic to the poset $P_{X} \cup\{a b c d\}$. The element abcd $\in \mathbf{L C M}(I)$ does not contribute a free module to the minimal free resolution and therefore $I$ is a dispersed rigid monomial ideal. Since removing the element abcd from $\mathbf{L C M}(I)$ produces a poset which is not a lattice, there is no finite atomic lattice less than $\mathbf{L C M}(I)$ appearing in the stratum 
$\mathcal{L}(5)_{(1,5,7,4,1)}$ which is the lcm-lattice of a rigid concentrated monomial ideal. We therefore cannot apply the combination of Theorem 3.2 and Theorem 2.1 to construct a poset resolution of $I$ supported on its lcmlattice. However, we can realize the minimal resolution of this ideal as a CW-poset resolution supported on $P_{X}$. Indeed, removing the element abcd does not change the homology of the order complex for intervals $(\hat{0}, m)$ where $m>$ abcd in $\mathbf{L C M}(I)$. We are currently studying the class of rigid ideals which are incomparable to concentrated rigid ideals in the same stratum.

Acknowledgments. We thank Ezra Miller and Irena Peeva for comments which helped improve an early draft of this paper and for their fundamental definitions and observations, without which this paper would not exist. Furthermore, we are indebted to the referee, whose suggestions helped create a more streamlined, precise, and cogent manuscript.

\section{REFERENCES}

1. D. Bayer, I. Peeva and B. Sturmfels, Monomial resolutions, Math. Res. Lett. 5 (1998), 31-46.

2. D. Bayer and B. Sturmfels, Cellular resolutions of monomial modules, J. reine agnew. Math. 502 (1998), 123-140.

3. A. Björner, Topological methods, in Topological methods. Handbook of combinatorics, Vol. 1, 2, 1819-1872, Elsevier, Amsterdam, 1995.

4. W. Bruns and J. Herzog, On multigraded resolutions, Math. Proc. Cambr. Philos. Soc. 118 (1995), 245-257.

5. T. Clark, Poset resolutions and lattice-linear monomial ideals, J. Algebra 323 (2010), 899-919.

6. D. Eisenbud, Commutative algebra. With a view toward algebraic geometry, Grad. Texts Math. 150, Springer-Verlag, New York, 1995.

7. Vesselin Gasharov, Irena Peeva and Volkmar Welker, The lcm-lattice in monomial resolutions, Math. Res. Lett. 6 (1999), 521-532.

8. Sonja Mapes, Finite atomic lattices and resolutions of monomial ideals, J. Algebra 379 (2013), 259-276.

9. E. Miller and B. Sturmfels, Combinatorial commutative algebra, Grad. Texts Math. 227, Springer Verlag, New York, 2004.

10. Ezra Miller, Planar graphs as minimal resolutions of trivariate monomial ideals, Doc. Math. 7 (2002), 43-90.

11. , personal communication. 
12. J. Munkres, Elements of algebraic topology, Addison-Wesley Publishing Company, Menlo Park, CA, 1984.

13. I. Peeva and M. Velasco, Frames and degenerations of monomial resolutions, Trans. Amer. Math. Soc. 363 (2011).

14. Jeffery Phan, Order properties of monomial ideals and their free resolutions, Ph.D. thesis, Columbia University, 2006.

15. M. Velasco, Minimal free resolutions that are not supported by a $C W$ complex, J. Algebra 319 (2008), 102-114.

16. D. Visscher, Minimal free resolutions of complete bipartite graph ideals, Comm. Alg. 34, (2006), 3761-3766.

Department of Mathematics and Statistics, Knott Hall 308, Loyola University of Maryland, 4501 North Charles Street, Baltimore, MD 21210 Email address: tbclark@loyola.edu

Department of Mathematics, University of Notre Dame, 255 Hurley, Notre DAME, IN 46556

Email address: smapes1@nd.edu 